\title{
Dental management of patients with inflammatory bowel disease
}

\author{
Aisha Mancheño Franch ${ }^{1}$, Yolanda Jiménez Soriano ${ }^{2}, M^{a}$ Gracia Sarrión Pérez $^{1}$ \\ ${ }^{1}$ Dentist. \\ ${ }^{2}$ Assistant Professor Doctor. Department of Stomatology. Medicine and dentistry faculty. Valencia University. \\ Correspondence: \\ C/ Prof. Dr Severo Ochoa 4, esc izda pta 4 \\ 46010 Valencia \\ E-mail: aishamancheno@gmail.com
}

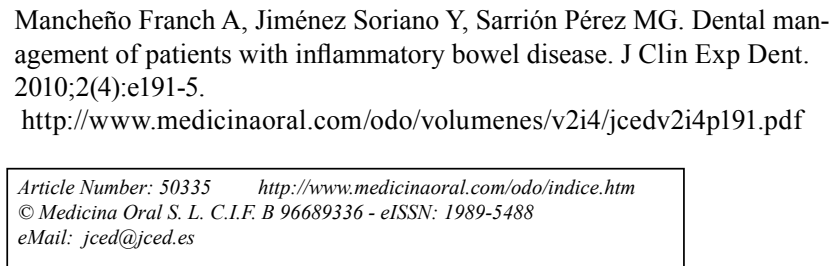

\begin{abstract}
Ulcerative colitis and Crohn's disease are the most common forms of inflammatory bowel disease (IBD), both of unknown aetiology. These conditions are characterised by the chronic and recurrent inflammation of different parts of the gastrointestinal tract, but while in $\mathrm{CD}$, chronic inflammation may affect any part of the gastrointestinal tract, in UC, mucosal inflammatory changes are confined to the colon. IBD is currently on the increase, and it is important for the dental professional to be familiar with the condition as patients with IBD may present oral manifestations of the underlying disease. Such manifestations of IBD may precede the onset of intestinal radiographic lesions by as much as a year, or even more. Treatments used to manage IBD can affect the delivery of routine dental care.
\end{abstract}

Key words: inflammatory bowel disease, Chroh's disease, ulcerative colitis, oral manifestations, pyostomatitis vegetans. 


\section{Introduction}

Inflammatory bowel diseases (IBD) include Crohn's disease (CD), ulcerative colitis (UC) and an ill-defined group of medical conditions known as indeterminate colitis. These are characterised by the chronic and recurrent inflammation of different parts of the gastrointestinal tract (1).

In $\mathrm{CD}$, chronic inflammation may affect any part of the gastrointestinal tract, whereas in UC, mucosal inflammatory changes are confined to the colon $(1,2)$. The term indeterminate colitis should be reserved only for those cases where colectomy has been performed and pathologists are unable to make a definitive diagnosis of either $\mathrm{CD}$ or UC after full examination, and the term $I B D$ type unclassified is suggested for patients in whom there is clinical and endoscopic evidence of chronic IBD affecting the colon, without small bowel involvement, and with no definitive histological or other evidence to favour either CD or UC (3).

The aetiology of IBD is unknown: it hasn't been established if there is a cause and effect relationship with a single agent that causes the disease's characteristics, or whether inherited molecular alterations are responsible. The currently more accepted etiopathogenic theory suggests that the intestinal inflammation is a consequence of an abnormal action of $\mathrm{T}$ lymphocytes upon the enterical bacterial flora in a genetically susceptible individual (4).

The onset of the disease occurs more often in the second or the third decade of life, but there also is another peak in the $60 \mathrm{~s}(5)$, without a clear predominance of gender (4).

The incidence, and consequently the prevalence, of IBD has been increasing of late. The prevalence of IBD is 1 in 10,000 people.

It is important for the dental professional to be familiar with the condition as patients with IBD may present oral manifestations of the underlying disease. Such manifestations of IBD may precede the onset of intestinal radiographic lesions by as much as a year, or even more. Treatments used to manage IBD can affect the delivery of routine dental care. (6).

\section{Objectives}

The aim of this article is to carry out a review of literature about IBD, and to examine the latest research concerning the oral manifestations and dental management of patients with this condition.

\section{Material and methods}

The subject-related research was carried out on the Pubmed-Medline database. As a research strategy we used different combinations of the following keywords: inflammatory bowel disease, Crohn's disease, ulcerative colitis, oral manifestations, oral complications, dental management, pyostomatitis vegetans.

We included articles published in both English and Spanish from 1999. We reviewed 17 articles.

\section{Results}

Ulcerative colitis (UC)

UC is an inflammatory disease of the bowel affecting the superficial lining mucosa in the rectum and the large intestine. The disease typically starts from the rectum and continues through the large bowel sparing the deeper layers of the intestinal wall. The small intestine is rarely affected (1).

Upon endoscopic examination of patients with UC, the intestinal mucosa presents friability, erythema, ulcerations, and pseudopolyps. Histological samples reveal significant lymphocytic infiltration of lamina propria, fine granularity, cryptic inflammation, and abscess (5).

Based on the extent of colonic involvement, UC can be classified into:

- ulcerative proctitis, when involvement is limited to the rectum

- left sided UC, when involvement is limited to a portion of the colorectum distal to the splenic flexure,

- extensive UC, when involvement extends proximal to the splenic flexure (3).

Signs and symptoms often worsen with withdrawal of feeding and include abdominal pain and distension, chronic diarrhea mixed with blood or mucous, weight loss, and fatigue (5). Mortality from UC is not significant, but the chronicity and periodic flare-ups, disease complications, as well as the cost and side effects of therapy, pose significant morbidity in those affected. Resection of colon often cures all systemic signs and symptoms, which may suggest the disease process is confined to the colon rather than involving the general immune system (1).

\section{Crohn's disease}

$\mathrm{CD}$ is a chronic, transmural inflammatory bowel disease that may affect any part of the bowel system including the oral cavity $(1,2)$. In contrast to ulcerative colitis, CD has a patchy rather than continuous distribution throughout the gut and is transmural with inflammation extending the entire thickness of the intestinal wall (1).

Upon endoscopic examination of patients with $\mathrm{CD}$, the diseased intestinal mucosa may present mucosal edema, thickening, obvious ulceration, cobblestoning, and pseudopolyps. Histological features of CD include infiltration of intestinal mucosa with neutrophils, lymphocytes, and mononuclear cells (5). Clusters of giant cells or noncaseating granulomas have been found in up to $60 \%$ of the cases (1).

Based on the bowel segment it affects, $\mathrm{CD}$ has been classified into:

- L1 ileitis, affecting ileum only,

- L2 colitis, affecting colon only,

- L3 ileocolic affecting both ileum and colon $(1,3)$ 


\begin{tabular}{|l|l|}
\hline Crohn's disease & Ulcerative colitis \\
\hline Any part of the gastrointestinal tract & Changes confined to the colon \\
Rare rectum involvement & Frequent rectum involvement \\
Common perianal disease & Rare perianal disease \\
Patchy inflammation & Continous inflammation \\
Transmural inflammation & Superficial lining mucosa inflammation \\
Common presence of fistulae and stenotic complication & Rare presence of fistulae and stenotic complication \\
Intraabdominal abscess & Rare presence of abscess \\
Deep ulcers, cobblestoning & Superficial ulcers, friability \\
Granulomas & Cryptic abscess \\
Lymphoid follicles & Crypts distortion \\
\hline
\end{tabular}

Table 1. Differences between Crohn's disease and ulcerative colitis.

- L4 upper, isolated upper disease (3).

Signs and symptoms of $\mathrm{CD}$ are variable and include abdominal cramps, diarrhea, melena, rectal bleeding, vomiting, poor appetite, fatigue and weight loss. The long-term progression of $\mathrm{CD}$ is unpredictable. Whereas in some patients, only the inflammatory component of the disease manifests, others may develop complications (1).

Table 1 provides a comparison summary of $\mathrm{CD}$ and CU.

The medical management of IBD includes the following (6):

- Antidiarrheal/antispasmodic agents (diphenoxylate and atropine),

- Anti-inflammatory agents (sulfasalazine or 5-aminosalicylates),

- Antibiotics (metronidazole),

- Corticosteroids (topical by enema and/or systemic),

Immunosuppressants (azothiaprine, 6-mercaptopurine).

Surgical intervention or colectomy is reserved for recalcitrant, severe cases of UC with significant hemorrhage, toxic megacolon, or perforation, and often brings about a cure.

\section{Oral manifestations of patient with IBD}

The clinical distinction between the oral manifestations of Crohn's disease and ulcerative colitis may be blurred with overlapping clinical features (7).

- Ulcerative colitis

Mucosal changes of UC in the oral cavity include stomatitis, glossitis, cheilitis, aphthous ulceration, and pyostomatitis vegetans (1), which represents a specific marker of ulcerative colitis even if the nature of this association is not clear (8).

Erosive temporo-mandibular joint disease secondary to ulcerative colitis-associated spondyloarthropathy, has also been reported $(7,9)$.

- Crohn's disease

Mucocutaneous changes of $\mathrm{CD}$ in the orofacial region include granulomatous changes of oral $\mathrm{CD}$ caused by direct extension of intestinal inflammation, or those of metastatic $\mathrm{CD}$ affecting a distant cutaneous site noncontiguous with the bowel. The prevalence of oral $C D$ varies between 0,5 and $80,0 \%$ and oral findings may precede onset of intestinal disease in up to $60 \%$ patients $(1,10)$.

Clinical manifestations of oral CD include specific and non-specific changes. Changes considered specific and pathogonomic for $\mathrm{CD}$ are cheilitis granulomatosa, cobblestoning of oral mucosa, mucosal tags and linear ulcerations, and hyperplastic folds of buccal vestibules $(1,2,11)$. Non-specific changes are angular cheilitis, aphthous stomatitis and pyostomatitis vegetans (1).

Dental erosion, halitosis, odynophagia, dysphagia, high prevalence of caries, granulomatous and autoimmunelike changes in minor salivary glands, and reduced salivation have also been reported (12).

Table 2 provides a summary of specific and nonspecific changes associated with IBD.

Orofacial granulomatosis

\begin{tabular}{|l|l|}
\hline \multicolumn{2}{|l|}{ Oral lesions in IBD } \\
\hline Specific & $\begin{array}{l}\text { Orofacial granulomatosis } \\
\text { Cobblestoning } \\
\text { Mucosal tags } \\
\text { Deep, linear ulcers with hyperplastic folds } \\
\text { Pyostomatitis vegetans }\end{array}$ \\
\hline Nonspecific & $\begin{array}{l}\text { Aphthous ulcers } \\
\text { Angular cheilitis } \\
\text { Labial/facial edema } \\
\text { Gingivitis } \\
\text { Gingival erythema/edema }\end{array}$ \\
\hline
\end{tabular}

Table 2. Oral lesions in IBD (11).

Oral granulomatosis is a common manifestation in patients with IBD and is typified by recurrent or persistent swelling of the lips, cheeks, gingivae, or oral mucosa with characteristic noncaseating granulomas on histologic examination (11). The lips are the most frequent 
site of involvement; the labial tissues demonstrate a nontender, persistent swelling that may involve one or both lips (13). Many patients with orofacial granulomatosis do eventually develop gastrointestinal disease consistent with Crohn's disease (11).

\section{Cobblestoning mucosa and mucosal tags}

Cobblestoning refers to nodular granulomatous swellings that result in a cobblestone appearance of the oral mucosa, particularly on the labial and buccal mucosa. Along with mucosal tags, cobblestoning is highly suggestive of CD (11).

Deep linear ulcers with hyperplastic folds

They are specifically associated with IBD (11). These ulcerations may cause pain on touch, or when eating acidic, spicy or hot foods. These ulcers, which are typically persistent, linear and deep, should not be confused with aphthous ulcers, which are shallow, round to oval shaped lesions that heal spontaneously in approximately seven to 14 days (7).

\section{Pyostomatitis vegetans}

Although IBD may precede the onset of oral or cutaneous lesions by months or years, sometimes the symptoms may be minimal and not sufficient to make an early diagnosis. In these cases the identification of PV could represent a reason to encourage diagnostic investigations intended to reveal subclinical intestinal diseases (8). Clinically PV is characterised by multiple pustules on an erythematous base. The friable pustules have a grey to yellow necrotic appearance; they erode and form shallow 'snail-track' ulcers which may affect all areas of oral mucosa, although the most commonly affected sites are the labial and buccal mucosae, hard and soft palate, gingivae and sulci, while the least affected sites are the tongue and floor of mouth. Vegetations in areas of erythema can be seen especially on the gingivae and palate. As vegetating lesions progress the mucosa may be thrown into verrucous folds, particularly the lesions of buccal and labial mucosae which are soft and oedematous. Pain is not prominent even when there is extensive oral involvement (14).

Microscopically, the lesions mimic the crypt abscesses of colonic lesions, without evidence of granulomatous inflammation. The oral submucosa shows edema with neutrophils, eosinophils and lymphocytes, while the epithelium shows spongiosis with neutrophilic and eosinophilic abscesses.

The primary objective in the treatment of PV must be identifying and/or controlling the associated bowel disease. Lesions frequently improve when the colitis is controlled; likewise, an exacerbation of colitis usually is followed by a similar flare in oral lesions.

Oral lesions are often treated effectively with topical corticosteroids in a gel or mouthwash formulation. However, systemic corticosteroids, dapsone and other immunosuppressive therapy may be suggested for mo- derate-to-severe symptomatic lesions in the oral cavity that are recalcitrant to topical therapy (15).

Aphthous ulcers

Aphthous ulcers are considered by many to be non-specific, as they can be seen in up to $20 \%$ of the general population; however aphtae are usually more extensive and persistent when associated with IBD (11). If a patient develops IBD during adulthood but has a history of recurrent aphthous ulcerations since adolescence, it is likely that the aphthous ulcers represent a coincident process that may be exacerbated by IBD or its management. For example, anti-inflammatory medications such as 5-aminosalicylates, the mainstay of IBD treatment, are excreted in saliva and are known to cause aphthous ulcers in some patients (6).

Aphthous ulcerations are a primary component of Behçet syndrome, which has also been implicated in inflammation of the large bowel (6), and also of Sweet's syndrome, which is a rare cutaneous manifestation of ulcerative colitis or Crohn's disease (16). In the past, it was believed that the association between an uncommon disorder like Sweet's syndrome and a relatively common disease like IBD was coincidental, but given the increasing number of case reports on Sweet's syndrome and IBD (IBD is now the third most common disease associated with Sweet's syndrome) (17), this is unlikely. The other factor supporting a true association between Sweet's syndrome and IBD is that Sweet's syndrome is from the same family of cutaneous disorders as erythema nodosum and pyoderma gangrenosum, suggesting a possible common pathogenesis (16).

\section{Other associations}

It is difficult to determine which oral manifestations are expressions of IBD, which represent preexisting and/ or coincidental findings, and which result directly from medical treatment of bowel disease.

Although candidosis is often associated with IBD patients, it probably represents either a reaction to the bacteriostatic effect of sulfasalazine or an impaired ability of neutrophils to kill this granuloma provoking organism, rather than a primary manifestation of the disorder. Likewise, some anti-inflammatory and sulfa-containing preparations used to manage IBD patients are reported to cause lichenoid drug reactions (6).

\section{Dental management of patients with IBD}

Patients with IBD are at increased risk of developing dental caries and oral infections. The causes of the increased incidence are multiple, but they appear to be related to either the patient's altered immune status or to diet. It is also important to recognise the risk of adrenal gland suppression in patients receiving corticosteroids to manage their IBD. It may be necessary to augment the steroid regimen during some dental treatments, especially for anxious patients in whom preoperative or 
postoperative pain management is difficult or when a complicated or stressful procedure is anticipated.

Oral inflammatory and granulomatous lesions associated with IBD may respond to topical steroid therapy but should not be used indefinitely due to the risk of mucosal atrophy and systemic absorption (6).

Dental management of patients with IBD should include the following:

- Frequent preventive and routine dental care to prevent destruction of hard and soft tissue.

- Evaluation of hypothalamic/pituitary/adrenal cortical function to determine the patient's ability to undergo extensive dental procedures.

- Avoid prescribing non-steroidal anti-inflammatory drugs (NSAID), as they can trigger a flare-up. The use of paracetamol is recommended, although it can also adversely affect patients.

- Early diagnosis and treatment of oral infections to enhance the gastroenterologist's ability to manage the IBD.

- Diagnosis (biopsy if necessary) and treatment of oral inflammatory, infectious, or granulomatous oral lesions (6).

\section{Conclusions}

Mucosal changes of UC in the oral cavity include stomatitis, glossitis, cheilitis, aphtous ulcerations, and pyostomatitis vegetans. The latter represents a specific marker of ulcerative colitis (8).

Macrocheilia, cobblestoning of oral mucosa, deep linear ulcers of buccal vestibules, and polypoid mucosal tags are considered specific and pathogonomic for CD $(1,2,11)$. Nonspecific changes include angular cheilitis, aphthous stomatitis and pyostomatitis vegetans (1).

In dental treatment of patients with IBD, it is important that they undergo frequent dental revisions and preventive care to avoid oral infections and hard and soft tissue destruction; it is also important to diagnose and treat all inflammatory, infectious or granulomatous oral lesions. We also have to avoid NSAID prescriptions and evaluate hypothalamic/pituitary/adrenal cortical functions.

\section{References}

1. Fatahzadeh M. Inflammatory bowel disease. Oral Surg Oral Med Oral Pathol Oral Radiol Endod. 2009;108:e1-10.

2. Lourenço SV, Hussein TP, Bologna SB, Sipahi AM, Nico MM. Oral manifestations of inflammatory bowel disease: a review based on the observation of six cases. J Eur Acad Dermatol Venereol. 2010;24:204-7.

3. Satsangi J, Silverberg MS, Vermeire S, Colombel JF. The Montreal classification of inflammatory bowel disease: controversies, consensus, and implications. Gut. 2006;55:749-53.

4. Sicilia B, Vicente R, Gomollón F. Epidemiology of inflammatory bowel disease: controversies in classical epidemiology. Acta Gastroenterol Latinoam. 2009;39:135-45.

5. Karlinger K, Györke T, Makö E, Mester A, Tarján Z. The epidemiology and the pathogenesis of inflammatory bowel disease. Eur $\mathbf{J}$ Radiol. 2000;35:154-67.
6. Siegel MA, Jacobson JJ. Inflammatory bowel diseases and the oral cavity. Oral Surg Oral Med Oral Pathol Oral Radiol Endod. 1999;87:12-4.

7. Daley TD, Armstrong JE. Oral manifestations of gastrointestinal diseases. Can J Gastroenterol. 2007;21:241-4.

8. Femiano F, Lanza A, Buonaiuto C, Perillo L, Dell'Ermo A, Cirillo N. Pyostomatitis vegetans: a review of the literature. Med Oral Patol Oral Cir Bucal. 2009;14:E114-7.

9. Chaudhry SI, Philpot NS, Odell EW, Challacombe SJ, Shirlaw PJ. Pyostomatitis vegetans associated with asymptomatic ulcerative colitis: a case report. Oral Surg Oral Med Oral Pathol Oral Radiol Endod. $1999 ; 87: 327-30$.

10. Lourenço SV, Boggio P, Bernardelli IM, Nico MM. Oral ulcers on the vestibular sulci. Diagnosis: oral Crohn's disease. Clin Exp Dermatol. 2006;31:735-6.

11. Galbraith SS, Drolet BA, Kugathasan S, Paller AS, Esterly NB. Asymptomatic inflammatory bowel disease presenting with mucocutaneous findings. Pediatrics. 2005;116:e439-44.

12. Katz J, Shenkman A, Stavropoulos F, Melzer E. Oral signs and symptoms in relation to disease activity and site of involvement in patients with inflammatory bowel disease. Oral Dis. 2003;9:34-40.

13. Ojha J, Cohen DM, Islam NM, Stewart CM, Katz J, Bhattacharyya I. Gingival involvement in Crohn disease. J Am Dent Assoc. 2007;138:1574-81; quiz 1614-5.

14. Hegarty AM, Barrett AW, Scully C. Pyostomatitis vegetans. Clin Exp Dermatol. 2004; 29:1-7.

15. DeRossi SS, Salazar G, Sarin J, Alawi F. Chronic lesions of the gingiva and mucosa. J Am Dent Assoc. 2007 ;138:1589-92.

16. Ali M, Duerksen DR. Ulcerative colitis and Sweet's syndrome: a case report and review of the literature. Can J Gastroenterol. 2008;22:296-8.

17. Cohen PR, Kurzrock R. Sweet's syndrome revisited: a review of disease concepts. Int J Dermatol. 2003;42:761-78. 